\title{
EFEITO CONTÁGIO NOS MERCADOS FINANCEIROS: LEVANTAMENTO SOBRE A PRODUÇÃO CIENTÍFICA NA BASE DO SCOPUS
}

\author{
Rodrigo Abbade da Silva1, Vinicius Girardi da Silveira, Raphael Silveira Amaro, Daniel \\ Arruda Coronel, Mygre Lopes da Silva, Vânia Medianeira Flores Costa
}

\section{RESUMO}

O presente estudo procurou contribuir para a temática de efeito contágio nos mercados financeiros, que ainda apresenta um conceito amplamente em discussão na literatura. Neste sentido, objetiva-se explorar a produção científica da temática efeito contágio e mercado financeiro, na área de administração, contabilidade e economia, por meio de uma pesquisa bibliométrica na base de dados Scopus, no período de 1999 a 2014. Ainda, verifica as características gerais das publicações na área e explica brevemente o conteúdo dos artigos mais citados. Foram encontrados 4.159 artigos com predomínio de trabalhos nos Estados Unidos, no Reino Unido e na Alemanha como os países com maior número de publicações enquanto o Brasil ocupa apenas a vigésima sétima posição no ranking referente ao número de publicações por países.

Palavras-chave: Efeito Contágion. Finanças. Bibliometria.

\section{THE CONTAGION EFFECT ON FINANCIAL MARKETS: A SURVEY ON THE SCIENTIFIC PRODUCTION IN THE SCOPUS DATABASE}

\begin{abstract}
The present study sought to contribute to the theme of contagion effect on financial markets, which still features a concept widely in discussion in the literature. In this sense, the goal is to explore the scientific production of the contagion effect thematic and financial market, in the area of administration, accounting and economics, through a Bibliometric research in the database Scopus, in the period from 1999 to 2014. Still, checks the general characteristics of the publications in the field and explains briefly the content of the cited articles. 4,159 articles were found with a predominance of papers in the United States, the United Kingdom and Germany as countries with the largest number of publications while Brazil occupies only the 27 th position in the ranking for the number of publications by country.
\end{abstract}

Keywords: Contagion Effect. Finance. Bibliometrics.

${ }^{1}$ Mestrando em administração pela UFSM, Bacharel em ciências econômicas pela UFSM 


\section{INTRODUÇÃO}

A globalização econômica e o avanço tecnológico têm intensificado as relações entre diferentes países nos últimos anos. Uma das consequências é que existe uma interligação cada vez mais acentuada e que os desequilíbrios iniciados em uma determinada região podem ser rapidamente transmitidos para outras regiões que estão geograficamente distantes, mesmo que possuam diferentes estruturas econômicas e não compartilhem laços econômicos significativos (PERICOLI; SBRACIA, 2003).

No início da década de 1990, elevou-se significativamente o nível de interação entre os mercados financeiros de diferentes países, devido a uma elevação dos fluxos de capitais internacionais, corroborando para o aumento da instabilidade econômica mundial (PEROBELLI; VIDAL; SECURATO, 2013). A ocorrência de desequilíbrios de caráter financeiro de um determinado país pode fazer com que seus investidores reestruturem seus portfólios, reconsiderando seus investimentos em diversos mercados, sem levarem em consideração os fundamentos macroeconômicos do país em questão (DORNBUSCH; PARK; CLAESSENS, 2000). Deste modo, pode ocorrer o efeito denominado de contágio, que representa a propagação de uma crise financeira de um determinado país para outros países e que não pode ser explicado pela interdependência no comércio ou por fatores macroeconômicos comuns entre esses mercados (STOVE; TJOSTHEIM; HUFTHAMMER, 2014).

A existência do efeito contágio, de um modo geral, pode provocar impactos nas decisões de investimentos em carteiras diversificadas internacionalmente, uma vez que a propagação das grandes quedas dos valores dos ativos financeiros de um país é rapidamente seguida por quedas de ativos financeiros de outros países (STOVE; TJOSTHEIM; HUFTHAMMER, 2014). Com isso, uma maior dependência entre os mercados financeiros, durante um período de crise, pode diminuir, ou até mesmo anular, o efeito da redução de risco proporcionado pelo processo de diversificação de carteiras de investimentos, conforme o que foi proposto, em 1952, por Markowitz (PEROBELLI; VIDAL; SECURATO, 2013).

Devido à importância de se estudar a existência do efeito contágio entre os países, diversos trabalhos acadêmicos e técnicos têm sido desenvolvidos nos últimos anos. De acordo com Vartanian (2012), a partir da segunda metade da década de 1990, em consequência da ocorrência de crises em mercados emergentes, amplia-se a discussão do efeito contágio na 
literatura existente. Em 2008, em virtude da crise financeira originada no mercado de hipotecas de alto risco dos Estados Unidos da América, emergiram diversos estudos evidenciando a existência do efeito contágio para outros segmentos do mercado financeiro, assim como para outros mercados financeiros internacionais (PRATES; CUNHA; LÉLIS, 2011).

Com o intuito de contribuir para a literatura existente, este trabalho se propõe a realizar um mapeamento dos artigos publicados sobre o efeito contágio, por meio de uma pesquisa bibliométrica, na base de dados Scopus, no período compreendido entre os anos de 1999 e 2014. Para realizar o levantamento sobre a produção científica, este trabalho utiliza as seguintes palavras-chave no campo de busca da base de dados: contagion effect e financial.

Diante do exposto, torna-se de grande valia o conhecimento de um estudo bibliométrico sobre o efeito contágio, uma vez que esta pesquisa pode servir como base para melhor compreender este tema complexo. Além disso, ao realizar um levantamento de dados, pode-se destacar o comportamento da evolução dos trabalhos científicos publicados ao longo dos anos, salientando tendências que permitam a orientação para pesquisas futuras sobre o tema.

Além desta introdução, o artigo está organizado da seguinte forma: a seção dois apresenta a revisão de literatura do efeito contágio em mercados financeiros; na seção três, são apresentados os procedimentos metodológicos; na seção quatro, expõe-se a análise e discussão dos resultados; e, por fim, são apresentadas as considerações finais.

\section{EFEITO CONTÁGIO EM MERCADOS FINANCEIROS}

O processo de globalização possibilitou um intenso intercâmbio cultural e econômico entre os países, fazendo com que o mercado acionário adquirisse um importante papel no cenário financeiro internacional. Tendo em vista as oportunidades acompanhadas por este processo, países, tanto desenvolvidos como emergentes, procuraram abrir suas economias a fim de receber novos investimentos. Esta mobilidade de capitais tornou o mercado acionário uma importante fonte de captação de recursos, permitindo o desenvolvimento de empresas e contribuindo para o progresso dos países.

Com a intensificação das relações entre os países, desequilíbrios iniciados em um país são rapidamente transmitidos para a economia de outros, muitas vezes de forma intensa e imprevisível, sendo esta situação caracterizada como crise financeira (PEROBELLI; VIDAL; SECURATO, 2013). Segundo Pasquariello (2008), próximo a períodos de crise financeira, grande parte dos mercados emergentes e alguns mercados desenvolvidos apresentam 
comportamentos inesperados, severos e muitas vezes excessivos. Além disso, esses mercados experimentam movimentos de queda nas ações e baixa liquidez, comprometendo a negociação de ativos.

De acordo com Perobelli, Vidal e Securato (2013), o advento de uma crise em determinado país pode levar os investidores a reestruturarem seus portfólios, reconsiderando seus investimentos em diversos mercados, sem levar em conta nem mesmo as diferenças existentes entre os fundamentos macroeconômicos desses mercados. Esse tipo de transmissão de choques pode dar origem ao efeito contágio, causado por fenômenos irracionais, como pânicos financeiros, comportamentos de efeito manada, perda de confiança, aumento da aversão ao risco, entre outros. Bekiros (2014) destaca que o termo contágio tornou-se conhecido a partir da análise das crises da década de 1990, quando choques originados em determinado mercado estenderam-se a vários mercados de outros países e dificilmente conseguiam ser explicados por mudanças nos fundamentos macroeconômicos.

Segundo Forbes e Rigobon (2002), há certa integração pré-existente entre as economias dos países. Durante o período de turbulência financeira, essa relação apresenta uma tendência a se mostrar mais intensa. Essa intensidade pode ser suficiente para promover quebras na estrutura previamente existente de transmissão de choques entre dois países. Quando isso acontece, os autores caracterizam o episódio como efeito contágio, ou seja, choques ocorridos em determinada economia contagiaram a economia de outro país, independentemente da situação dos fundamentos macroeconômicos dos dois países em questão. Por outro lado, quando há um aumento normal na intensidade das relações, este pode ser classificado como interdependência, oriunda das relações econômicas preexistentes entre os países (PEROBELLI; VIDAL; SECURATO, 2013).

Para Lobão (1999), a teoria clássica se inclinou a analisar os efeitos de contágio entre as economias mundiais, identificando dois possíveis canais de transmissão: 1) canais fundamentais de propagação das instabilidades externas, os quais são é formados pelos canais financeiros e comerciais e estão relacionados ao ambiente de interdependência entre as economias; 2) contágio puro, quando as causas fundamentais não são suficientes para explicar a totalidade dos efeitos de contágio, em que as variáveis econômicas observáveis não permitem analisar a totalidade dos movimentos dos mercados financeiros entre as economias. Neste sentido, faz-se necessária a análise do contágio puro, que considera as causas que não estão relacionadas às causas fundamentais. 
Segundo Macedo, Casa Nova e Almeida (2010), o canal financeiro de propagação das crises relaciona-se ao comportamento dos investidores internacionais, que, diante de instabilidades, analisam a conjuntura econômica, podendo haver uma reversão em suas expectativas e leválos a alterarem suas posições em investimentos, o que compromete a entrada dos fluxos de divisas para algumas economias. Este canal de contágio pode ser dividido entre canal direto e indireto. O canal direto corresponde aos efeitos nas relações de investimentos e financiamentos existentes nos países diretamente envolvidos nas instabilidades econômicas. $\mathrm{O}$ canal indireto corresponde aos efeitos ocorridos em um terceiro país de mesmo grau de risco, mas que não está envolvido nas instabilidades. Geralmente os efeitos são resultado da existência de um credor comum.

Ainda, segundo Macedo, Casa Nova e Almeida (2010), o contágio por meio do canal financeiro ocorre de maneira muito mais rápida do que pelo canal comercial. Ao se confirmar um estado de instabilidade econômica em um país, a decisão de investimento especulativo (investimento de curto e médio prazo, realizado nas bolsas de valores) neste país e em outras economias semelhantes, é reajustada e, na maioria de vezes, os investimentos são direcionados a outros mercados determinados seguros por estes. No canal comercial, existe uma defasagem temporal para se efetivar o contágio, por se tratar de um setor com decisões de investimento de longo prazo e por caracterizar um investimento produtivo de menor liquidez.

Já quanto ao contágio puro, Perobelli; Vidal; Securato( 2013) explicam que este ocorre devido ao comportamento dos investidores nos mercados internacionais, não mantendo ligações com as características comerciais ou financeiras com os principais fatores ligados ao contágio puro, referindo-se à imitação entre os agentes ou a problemas de informação advindos das imperfeições de mercado.

Ainda segundo Perobelli, Vidal e Securato (2013), o comportamento de imitação por parte dos agentes, conhecido na literatura por herding behavior, pode contribuir para o contágio de crises caso os investidores internacionais se sintam motivados a adotar tal atitude. Tal comportamento se baseia em pressupostos quanto à capacidade de recepção das informações e dos respectivos tratamentos dados a elas por parte dos investidores. Em determinados contextos, as informações privadas a cada agente de mercado podem vir a ser abandonadas e levar a uma convergência de aceitação de informações uniformes, resultando na imitação por parte dos investidores. 
De acordo com Guidugli (2005), os trabalhos sobre efeito contágio fazem parte de um conjunto extenso de estudos sobre os impactos e transmissão de choques internacionais,

podendo ser agrupados em três gerações distintas. Como descrito por Perobelli, Vidal e Securato (2013), na primeira geração de estudos sobre a transmissão de choques internacionalmente, os autores analisam primordialmente os fundamentos macroeconômicos e suas mudanças, que deixariam um país vulnerável a choques externos, justificando assim a propagação das crises e seu impacto distinto em cada país. Na segunda geração de estudos sobre crises financeiras, os autores analisam o impacto das expectativas dos investidores na propagação das crises, assim como comportamentos irracionais que intensificariam o efeito dos choques. Já os estudos da terceira geração evidenciam a existência do efeito contágio dos choques e formas de mensurá-lo, reconhecendo a existência de certa inter-relação preexistente entre as economias e o contágio como uma quebra nesta estrutura de relações. Nesta última geração de estudos, os autores consideram que a propagação de choques a partir de mudanças nos fundamentos macroeconômicos vem da relação de interdependência entre as economias e consiste numa situação esperada. O efeito contágio seria então constituído por uma quebra estrutural nas relações que não pode ser explicada pelos fundamentos.

\section{PROCEDIMENTOS METODOLÓGICOS}

Utilizou-se uma técnica indireta de tratamento de dados. A partir do levantamento bibliográfico, foram elaboradas as análises do estudo. Quanto à sua natureza, a pesquisa apresenta um caráter aplicado a fim de adquirir conhecimentos para aplicação em um tema específico (LAKATOS; MARCONI, 2010). Além disso, a pesquisa caracteriza-se pelo cunho exploratório, uma vez que busca estabelecer maior familiaridade e percepção com o tema (GIL, 2002). Deste modo, foi realizado um levantamento de estudos que abordaram questões relacionadas a efeito contágio nos mercados financeiros mundiais.

Referente ao procedimento metodológico, o estudo foi desenvolvido por meio de uma pesquisa bibliométrica, utilizando as palavras contagion effect e financial na base de dados Scopus, no período de 1999 a 2014, cuja data inicial marca o período de maior intensificação na globalização e integração instantânea da informação (CASTELLS, 1999) e o último período é o mais recente disponível na base de dados utilizada. A bibliometria é uma atividade científica ou técnica que aplica métodos estatísticos e matemáticos sobre o conjunto de publicações e referências bibliográficas. Ainda, essa técnica permite o conhecimento do 
estágio de uma pesquisa, determinando em que estágio um tema encontra-se em uma determinada área (ROSTAING, 1996; MACEDO; CASA NOVA; DE ALMEIDA, 2010). A presente pesquisa foi dividida em três etapas, conforme Figura 1:

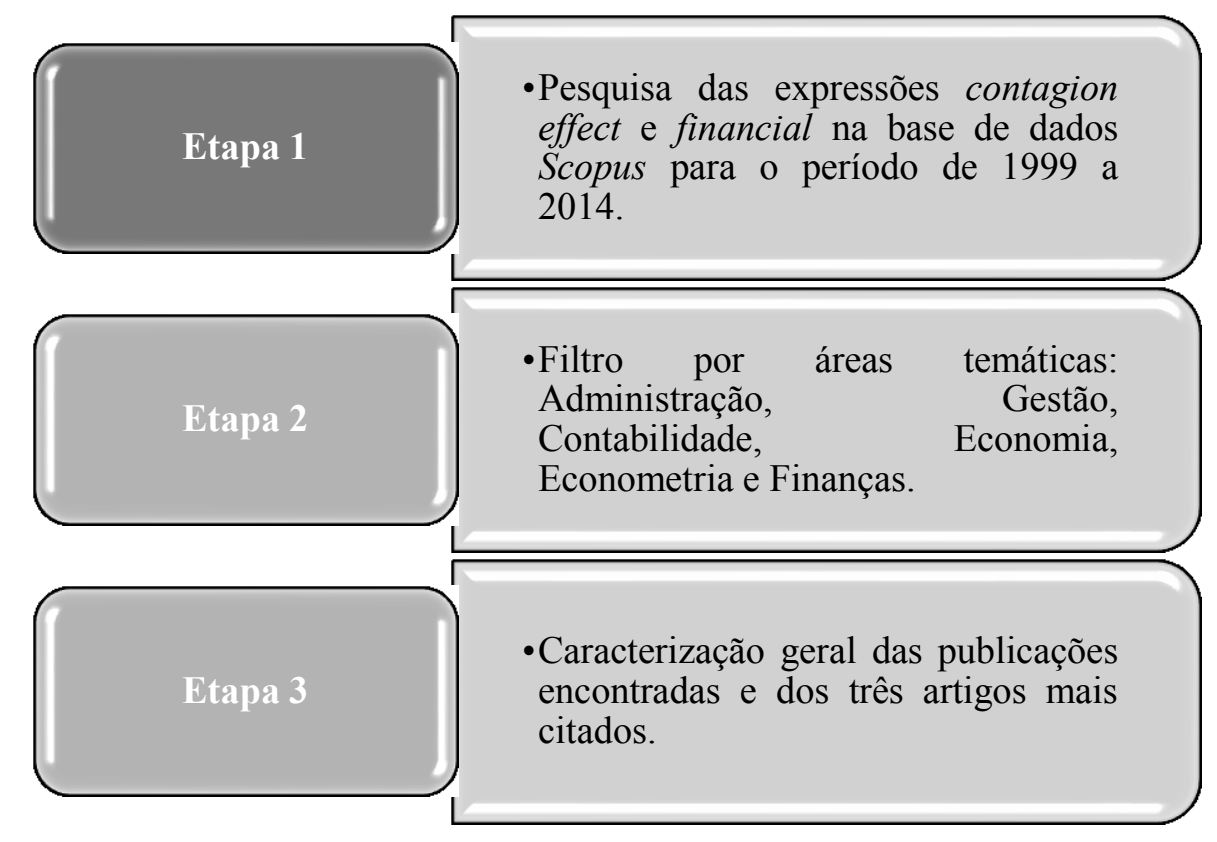

Figura 1 - Etapas da pesquisa

Fonte: Elaborado pelos autores

Diante desse contexto, após a pesquisa dos dois tópicos realizadas na etapa 1 e no filtro pela área temática do escopo do trabalho realizado na etapa 2, a etapa posterior procurou caracterizar os trabalhos encontrados. Portanto, por meio da etapa 3, foi feita uma caracterização geral dos trabalhos encontrados, isto é, informações sobre ano das publicações, países, instituições, periódicos e autores dos artigos encontrados. Posteriormente, foi realizada uma análise com mais acuidade dos três artigos com maior número de citações no período de análise.

\section{ANÁLISE E DISCUSSÃO DOS RESULTADOS}

\subsection{Caracterização geral das publicações}

A pesquisa na plataforma Scopus a partir da inserção dos tópicos contagion effect e financial apresentou, por meio de um filtro dentro das áreas de interesse de estudo, ou seja, administração, gestão, contabilidade, economia, econometria e finanças, o número de 4.159 artigos. Desta forma, foram realizadas análises sobre os 4.159 artigos encontrados dentro das áreas temáticas pré-determinadas, caracterizando a evolução quantitativa da produção 
científica entre efeito contágio no mercado financeiro mundial, no período de 1999 a 2014, por meio dos seguintes aspectos: ano, periódico, autores, instituições e países.

De acordo com a Figura 2, verifica-se a evolução do número de publicações de 1999 a 2014.

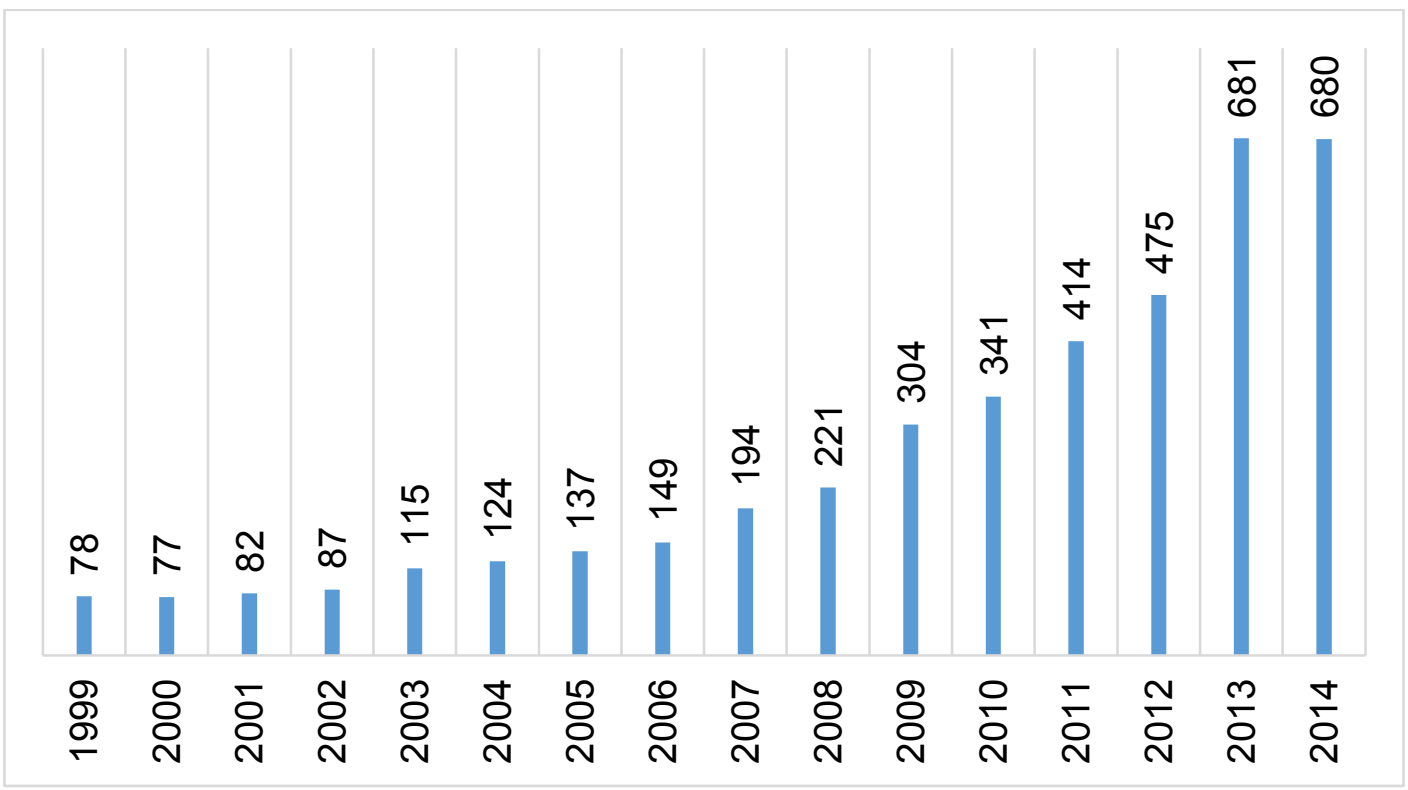

Figura 2 - Ano das publicações

Fonte: Elaborado pelos autores

Mediante análise temporal, verificou-se uma elevação das publicações nos anos de 2009 e, principalmente de 2013, quando foram alcançadas mais de 680 publicações sobre o tema. Esse resultado pode estar relacionado com os efeitos da Crise do Subprime iniciada em 2007, tornando-se sistêmica a partir de 2008 (TORRES FILHO, 2008; BRAGA, 2009). A crise deu maior visibilidade para os assuntos relacionados a efeito contágio no mercado financeiro mundial, devido à retração da demanda por commodities e à diminuição global do valor monetário negociado em mercados financeiros.

A Figura 3 demonstra os periódicos com maior número de publicações dentro dessa temática. 


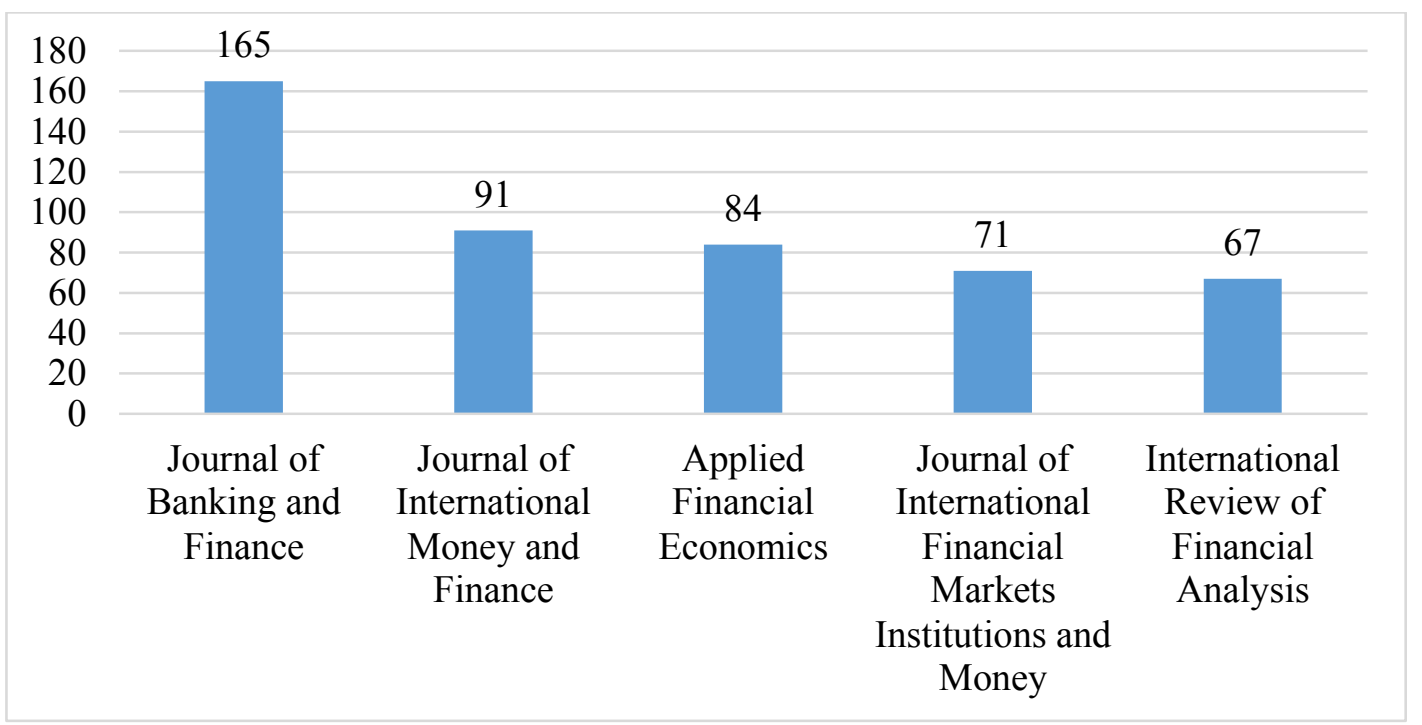

Figura 3 - Periódicos das publicações

Fonte: Elaborado pelos autores

Ainda conforme a Figura 3, os dois periódicos com maior número de publicações são o Journal of Banking and Finance e o Journal of International Money and Finance, com $165 \mathrm{e}$ 91 artigos, respectivamente, publicados no período. Por lidar com questões de desenvolvimento econômico, o escopo das revistas acadêmicas está adequado às temáticas que englobam aspectos do efeito contágio no mercado financeiro mundial. Os outros três periódicos, que apresentaram em média 74 publicações no período, estão relacionados às grandes áreas de pesquisa que englobam os tópicos abordados.

A Figura 4 apresenta a distribuição por publicações em relação aos autores.

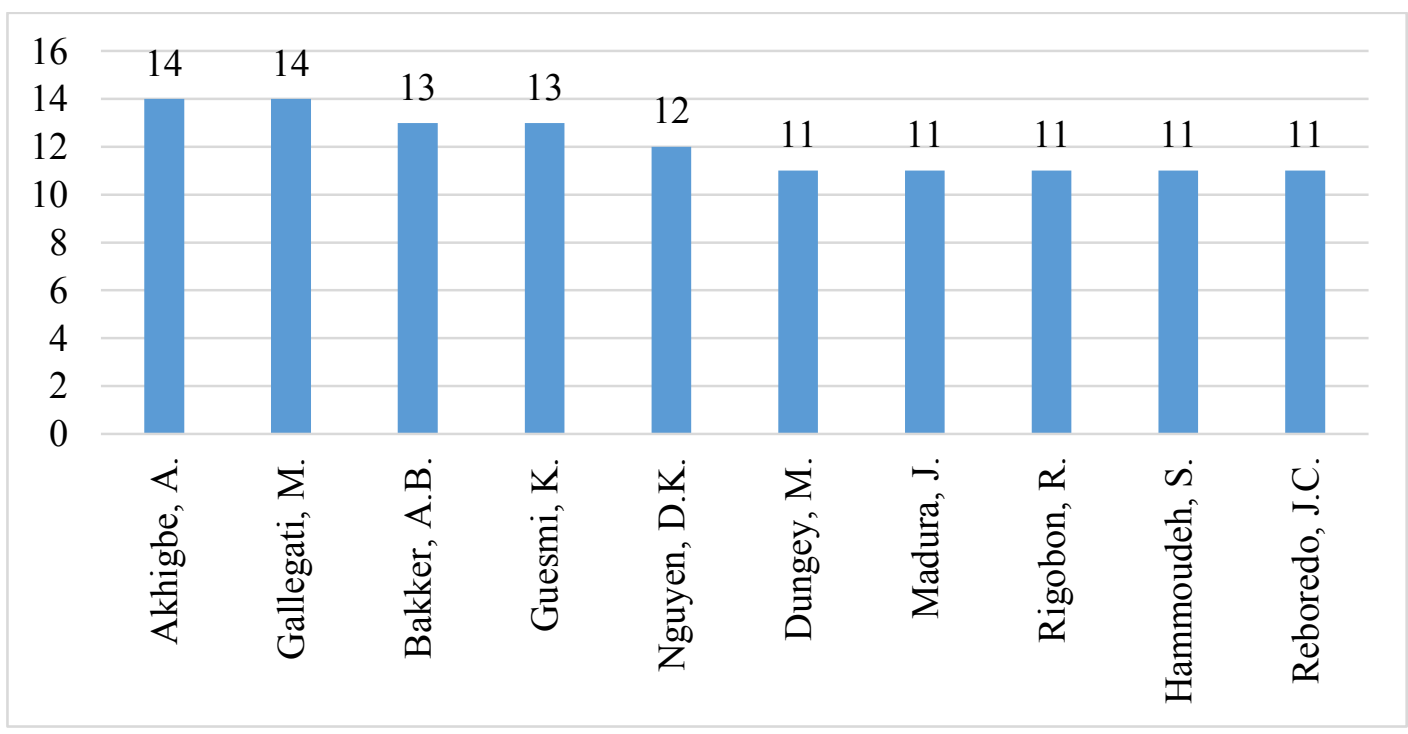

Figura 4 - Autores das publicações

Fonte: Elaborado pelos autores

Verifica-se distribuição relativamente homogênea entre os dez principais autores da temática em questão. Pode-se destacar os autores Aigbe Akhigbe, Mauro Gallegati, Bakker, Arnold B. 
e Guesmi, K., os quais apresentaram, respectivamente, quatorze, quatorze, treze e treze artigos publicados sobre a temática de 1999 a 2014. Aigbe Akhigbe, é Ph.D. pela University of Houston em finanças e faz parte do corpo docente da University of Akron. Mauro Gallegati é $\mathrm{PhD}$ pela Marche Polytechnic University em economia e tem suas contribuições teóricas mais significativas no campo da complexidade econômica (flutuações de negócios, dinâmica não linear, modelos de fragilidade financeira e interações entre agentes heterogênicos). Atualmente, é professor pela Marche Polytechnic University. Bakker, Arnold B. é professor da Erasmus University Rotterdam, além de pesquisar questões de psicologia, administração, gestão e contabilidade. Guesmi, Khaled é PhD. Pela University of Paris West La Défense em finanças e professor de finanças em IPAG Business School e pesquisa nas áreas de economia, econometria e finanças, negócios, gestão e contabilidade (SCOPUS, 2015).

A Figura 5 apresenta o número de publicações no período analisado conforme as instituições responsáveis/financiadoras por/ de cada um dos trabalhos.

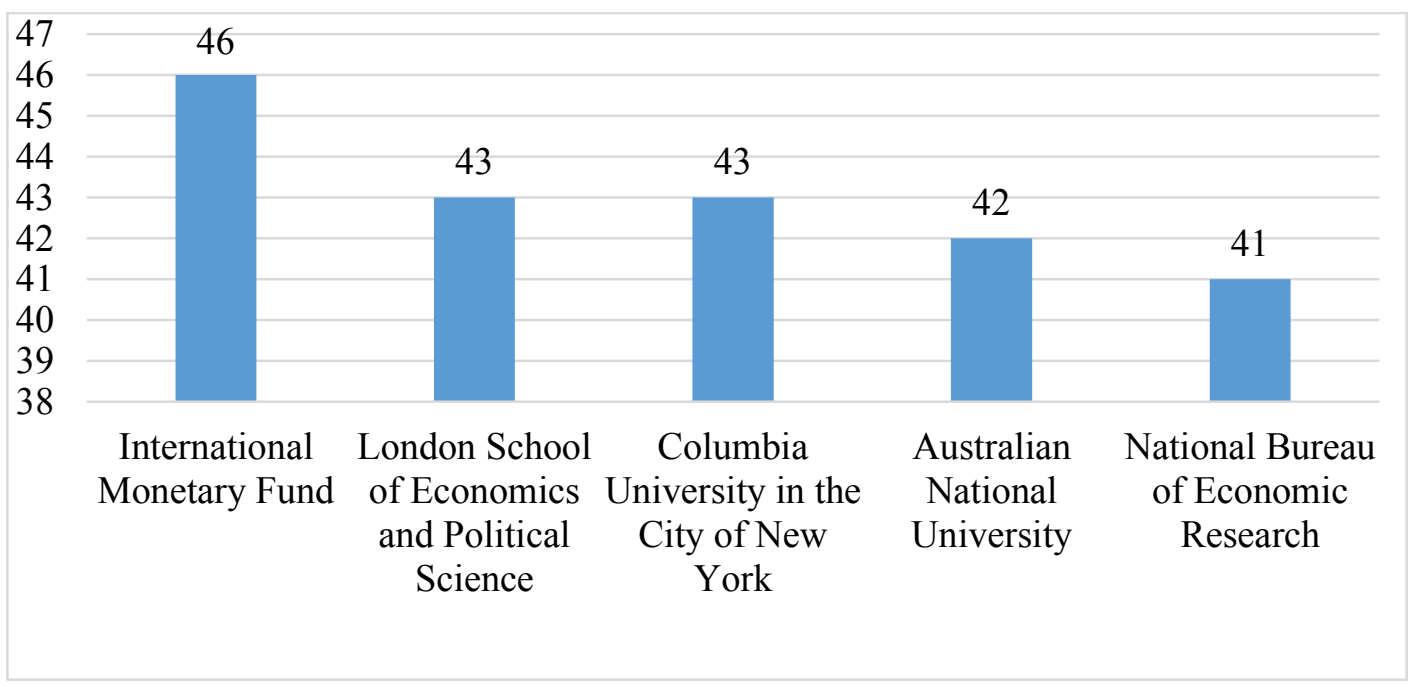

Figura 5 - Instituições das publicações

Fonte: Elaborado pelos autores

A partir da Figura 5, observa-se que as cinco instituições com maior número de publicações são dos Estados Unidos da América (EUA), do Reino Unido e da Austrália, fazendo parte dos continentes que, de uma forma geral, dominam os campos de pesquisas científicas dentro das áreas temáticas do presente estudo, isto é, Europa, América do Norte e Oceania. Além disso, é válido destacar que o Fundo Monetário Internacional - FMI foi a única instituição não acadêmica que apareceu nessa lista das instituições de maiores publicadores, tendo em vista ser uma instituição financeira com pesquisas dentro dessa área. 
Por último, com relação a essa caracterização inicial, a Figura 6 apresenta o número de publicações pelos principais países, os quais representam cerca de $75,0 \%$ do total de publicações no período de 1999 a 2014.

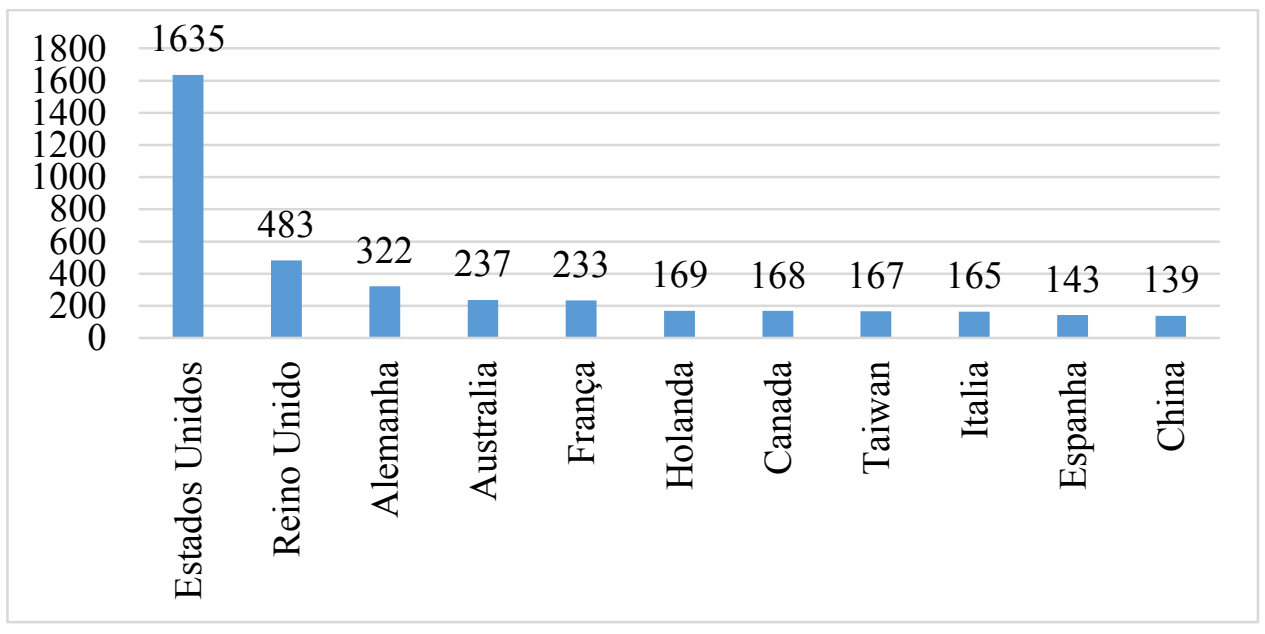

Figura 6 - Países das publicações

Fonte: Elaborado pelos autores

Verifica-se, por meio da Figura 6, um amplo domínio das publicações de origem americana. Além disso, Reino Unido e Alemanha, juntamente com os Estados Unidos, apresentam cerca de $45 \%$ do número de publicações encontradas, demonstrando o domínio dessas três regiões dentro do tópico pesquisado. Por fim, é válido destacar a posição do Brasil, com 39 publicações e ocupando o vigésimo sétimo lugar no ranking de publicação por países. Embora tem-se os 5 autores brasileiros que mais publicaram (número artigos), nesse período foram: Benjamin Miranda Tabak (38); Paulo Sérgio Ceretta (25); Charbel José Chiappetta Jabbour (18); Marcelo Brutti Righi (16) e Ricardo Pereira Câmara Leal (16). Cabe salientar que as pesquisas acadêmicas de brasileiros nessa área apresentam-se de forma incipiente dentro de um contexto global.

\subsection{Caracterização das publicações mais citadas}

De forma geral, o número de citações demonstra o quanto o artigo pode ser relevante para a evolução de uma determinaria área de estudos, tendo em vista que outros autores estão utilizando um mesmo artigo para contribuir para o estado da arte de um tema. Dessa forma, a Figura 7 demonstra os artigos com maiores números de citações na pesquisa realizada.

\begin{tabular}{|c|c|}
\hline Artigos mais citados & $\begin{array}{c}\mathrm{N}^{\mathrm{o}} \mathrm{de} \\
\text { citações }\end{array}$ \\
\hline $\begin{array}{c}\text { The twin crises: The causes of banking and balance-of-payments problems } \\
\text { Autor(es): Kaminsky G.L., Reinhart C.M. }\end{array}$ & 1060 \\
Publicado em: American Economic Review 89 (3), p. 473-500, 1999 & 939 \\
\hline No contagion, only interdependence: Measuring stock market comovements & \\
\hline
\end{tabular}




\begin{tabular}{|c|c|}
\hline $\begin{array}{c}\text { Autor(es): Forbes K.J., Rigobon R. } \\
\text { Publicado em: Journal of Finance 57 (5), p. 2223-2261,2002 }\end{array}$ & \\
\hline $\begin{array}{c}\text { Financial contagion } \\
\text { Autor(es): Allen F., Gale D. } \\
\text { Publicado em: Journal of Political Economy } 108 \text { (1), p. 1-33, } 2000\end{array}$ & 621 \\
\hline $\begin{array}{l}\text { Investor psychology and asset pricing } \\
\text { Autor(es): Hirshleifer D. } \\
\text { Publicado em: Journal of Finance 56 (4), p. 1533-1597, } 2001\end{array}$ & 460 \\
\hline $\begin{array}{c}\text { Overconfidence and speculative bubbles } \\
\text { Autor(es): Scheinkman J.A., Xiong W. } \\
\text { Publicado em: Journal of Political Economy } 111(6), \text { p. 1183-1219, } 2003\end{array}$ & 356 \\
\hline $\begin{array}{l}\text { A New Approach to Measuring Financial Contagion } \\
\text { Autor(es): Bae K.H., Karolyi G.A., Stulz R.M. } \\
\text { Publicado em: Review of Financial Studies 16 (3), p. 717-763, } 2003\end{array}$ & 286 \\
\hline $\begin{array}{c}\text { Towards a model of work engagement } \\
\text { Autor(es): Bakker A.B., Demerouti E. } \\
\text { Publicado em: Career Development International } 13 \text { (3), p. 209-223, } 2008\end{array}$ & 274 \\
\hline $\begin{array}{c}\text { Market Integration and Contagion } \\
\text { Autor(es): Bekaert G., Harvey C.R., Ng A. } \\
\text { Publicado em: Journal of Business } 78 \text { (1), p. 39-69, } 2005\end{array}$ & 272 \\
\hline $\begin{array}{l}\text { Work engagement and financial returns: A diary study on the role of job and personal } \\
\text { resources } \\
\text { Autor(es): Xanthopoulou D., Bakker A.B., Demerouti E., Schaufeli W.B. } \\
\text { Publicado em: Journal of Occupational and Organizational Psychology } 82 \text { (1), p. 183- } \\
200,2009\end{array}$ & 233 \\
\hline $\begin{array}{l}\text { The external wealth of nations: Measures of foreign assets and liabilities for industrial } \\
\text { and developing countries } \\
\text { Autor(es): Lane P.R., Milesi-Ferretti G.M. } \\
\text { Publicado em: Journal of International Economics 55 (2), p. 263-294, } 2001\end{array}$ & 210 \\
\hline
\end{tabular}

Figura 7 - Artigos com maiores números de citações

Fonte: Elaborado pelos autores

Nesta perspectiva, observa-se que, entre todos os trabalhos nesta temática, o estudo de Kaminsky e Reinhart (1999) é o que apresenta o maior número de citações, totalizando 1060. A pesquisa tem como objetivo preencher a lacuna de estudos de efeito contágio sobre moeda e bancos em episódios de crises para diversos setores industriais e países em desenvolvimento. Para isso, é aplicado o modelo econométrico Threshold, no período de 1970 até 1995, o qual contempla 76 crises econômicas e 26 crises no sistema bancário, bem como a crise gêmea na Ásia em 1997. Para isso, fez-se uso de 16 indicadores macroeconômicos.

Com isso, os autores descobriram que os típicos problemas do setor bancário precedem a ocorrência das crises, ou seja, a ocorrência das crises depende da ocorrência de crises no setor bancário, ativando uma viciosa espiral; liberalização financeira frequentemente precede crises bancárias. A anatomia destes episódios sugere que crises ocorrem quando a economia entra em recessão, seguindo um prolongado boom na atividade econômica, o qual foi alimentado por crédito, influxos de capital, e acompanhado por uma moeda sobrevalorizada. 
O trabalho de Forbes e Rigobon (2002), com 939 citações, o segundo mais citado, tem como objetivo mostrar que inferências com base no coeficiente de correlação condicional podem ser extremamente enganosas. Um simples ajuste para heterocedasticidade pode reverter o que parecem ser conclusões diretas sobre a existência de contágio durante crises cambiais recentes. Desta forma, faz-se uso de testes de testes de heterocedasticidade para polarizar testes de contágio com base em coeficientes de correlação.

Conforme os autores, os coeficientes de correlação estão sujeitos à volatilidade do mercado. Sob determinados pressupostos, é possível ajustar para este viés. Usando este ajustamento, não havia praticamente nenhum aumento nos coeficientes de correlação incondicionais, ou seja, nenhum contágio durante a crise de 1997, na Ásia, a de 1994, com a desvalorização mexicana, e a de 1987, no Crash do mercado dos Estados Unidos da América. Além disso, existe um elevado nível de comovimento entre os mercados em todos os períodos, o que os autores preferem denominar de interdependência.

O estudo de Allen e Gale (2000), com 621 citações, tem como objetivo central providenciar alguma fundamentação microeconômica para o efeito contágio. Para isso, as análises são realizadas para a crise financeira na Ásia por meio de diversos estudos teóricos discutidos sobre o efeito contágio em mercados financeiros atrás do uso de indicadores de liquidez bancária.

Com isso, descobriram que há pouca preferência por liquidez em mercados financeiros localizados em regiões que podem ser atingidas pelo efeito contágio. A possibilidade do contágio depende fortemente da plenitude da estrutura inter-regional das reivindicações. Além disso, estruturas de reinvindicações completas se mostraram mais eficientes que as incompletas.

O estudo de Hirshleifer (2001) é a quarta pesquisa mais citada no que se refere à temática de efeito contágio no mercado financeiro, com 460 citações. O artigo avalia a teoria e as evidências sobre a psicologia do investidor como determinante dos preços dos ativos. Esta questão está no centro de um grande debate em finanças abrangendo as duas últimas décadas. Nos últimos anos, economistas especializados em finanças têm dado maior atenção às explicações associadas à hipótese de que os humanos são racionais imperfeitos, ou seja, não têm conhecimento pleno de todas as informações de mercado. Para isso, o autor aplica e analisa um modelo CAPM (Capital Asset Pricing Model) de acordo com paradigmas da psicologia e estudos realizados relacionados à temática. 
Assim, as expectativas dos agentes econômicos mudam à medida que os agentes apreendem algo novo. Desta forma, sobre situações iguais, em tempos diferentes, os agentes podem ter reações diferentes, o que aumenta a complexidade das previsões econômicas quando algum efeito age sobre ela. Com isso, uma de suas principais contribuições é explicar a necessidade de que novas análises ou teorias atentem a esses comportamentos dos agentes, o que fomenta a nova corrente do pensamento em finanças, denominada de finanças comportamentais.

O trabalho de Scheinkman e Xiong (2003) é o quinto mais citado, com 356 citações. A pesquisa avalia o comportamento dos preços dos ativos, o volume de negócios e a volatilidade dos preços durante episódios de bolhas de preços de ativos. Para isso, fazem uso de um modelo econométrico de equilíbrio contínuo de tempo em que o excesso de confiança gera divergências entre os agentes quanto aos fundamentos dos ativos utilizando dados de diferentes estudos e confrontando seus respectivos resultados.

Desta forma, como agentes costumam pagar preços que excedem a sua própria valorização dos dividendos futuros, porque eles acreditam que, no futuro, eles vão encontrar um comprador disposto a pagar ainda mais, isso provoca um significativo componente da bolha de preços dos ativos, mesmo quando pequenas diferenças de crenças são suficientes para gerar um comércio. Todavia, em equilíbrio, as bolhas são acompanhadas por grande volume de negociação e de alta volatilidade dos preços. Com isso, percebe-se que, enquanto o imposto de Tobin pode reduzir substancialmente as especulações nas negociações, quando os custos de transação são pequenos, ele tem apenas um impacto limitado sobre o tamanho da bolha ou da volatilidade do preço.

A partir de uma análise das publicações mais citadas e dos trabalhos encontrados de uma forma geral na pesquisa do presente artigo, verifica-se que o efeito contágio no mercado financeiro relaciona-se de forma bastante comum dentro da literatura acadêmica, porém, em alguns casos por razões diferentes. Isso demonstra que já se encontram avançadas as pesquisas nesta temática e que a maioria dos trabalhos encontrados tratam de forma mais específica o assunto. Outras pesquisas, em menor número, abordam o efeito contágio como resultado de alterações nas expectativas ou no comportamento humano, como na linha de pesquisa de finanças comportamentais.

\section{CONSIDERAÇÕES FINAIS}

Ao relacionar os estudos de efeito contágio no mercado financeiro na base de dados Scopus, no período de 1999 a 2014, o presente artigo procurou retratar a importância do conceito de 
efeito contágio. Esse conceito demonstra o aumento da probabilidade condicional de ocorrer crise em um país, quando ocorre crise em outro país, e a relevância do sistema bancário ou sistema financeiro na ocorrência de crises, como canal de transbordamento das crises econômicas mundiais.

Quanto às características gerais dos 4.159 artigos encontrados por meio da pesquisa, verificase a predominância de artigos publicados nos anos de 2009 a 2013. Além disso, nota-se que o Journal of Banking and Finance e Journal ofInternational Money and Finance, o Fundo Monetário Internacional e a London School of Economics and Policies Science são os principais periódicos e instituições mais relevantes dentro da pesquisa realizada, respectivamente. Ademais, é válido destacar o predomínio de Estados Unidos, Reino Unido e Alemanha como os países com maiores números de publicações enquanto o Brasil ocupa a vigésima sétima posição no ranking referente a número de publicações por países.

Relativamente ao conteúdo dos artigos encontrados e, principalmente, àqueles com maior número de citações, verifica-se que o estado da arte na temática efeito contágio no mercado financeiro está consolidado. O que predomina são trabalhos de canal de transmissão das crises econômicas ou financeiras que tratam dos tópicos por meio de uma análise profunda.

Contudo este trabalho não deve ser considerado conclusivo, tendo em mente que investigações com maior profundidade podem apresentar resultados divergentes dos resultados alcançados. Assim, como limitações, este estudo apresenta a abordagem de certa forma simplista e com pouca profundidade e acuidade do método bibliométrico, além de a pesquisa ser realizada por uma única base de dados. Sugere-se, para trabalhos futuros, aprofundar este estudo, utilizando-se de tópicos emergentes e identificando as suas principais implicações bem como da utilização de outras bases de dados.

\section{REFERÊNCIAS}

ALLEN, F.; GALE, D. Financial contagion. Journal of political economy, v. 108, n. 1, p. 1$33,2000$.

BAE, K.-H.; KAROLYI, G. A.; STULZ, R. M. A new approach to measuring financial contagion. Review of Financial studies, v. 16, n. 3, p. 717-763, 2003.

BAKKER, A. B.; DEMEROUTI, E. Towards a model of work engagement. Career development international, v. 13, n. 3, p. 209-223, 2008.

BEKAERT, G.; HARVEY, C. R. Market integration and contagion. [s.1: s.n.].

BEKIROS, S. D. Contagion, decoupling and the spillover effects of the US financial crisis: 
Evidence from the BRIC markets. International Review of Financial Analysis, v. 33, p. 5869, maio 2014.

BRAGA, J. C. Crise sistêmica da financeirização e a incerteza das mudanças. estudos avançados, v. 23, n. 65, p. 89, 2009.

CASTELLS, M. A rede e o ser. São Paulo, SP, Brasil.: Paz e Terra, 1999.

DORNBUSCH, R.; PARK, Y. C.; CLAESSENS, S. Contagion: Understanding How It Spreads. The World Bank Research Observer, v. 15, n. 2, p. 177-197, 1 ago. 2000.

FORBES, K. J.; RIGOBON, R. No Contagion, Only Interdependence: Measuring Stock Market Comovements. The Journal of Finance, v. 57, n. 5, p. 2223-2261, 2002.

GIL, A. C. Como elaborar projetos de pesquisa. São Paulo, v. 5, p. 61, 2002.

GUIDUGLI, S. T. Análise multivariada do efeito contágio no episódio de ataque especulativo e crise cambial envolvendo o Brasil, a Rússia ea Argentina no período de 1998-99. [s.1: s.n.].

HIRSHLEIFER, D. Investor psychology and asset pricing. Journal of finance, p. 1533-1597, 2001.

KAMINSKY, G. L.; REINHART, C. M. The twin crises: the causes of banking and balanceof-payments problems. American economic review, p. 473-500, 1999.

LAKATOS, E. M.; MARCONI, M. DE A. Fundamentos da metodologia científica. In: Fundamentos da metodologia científica. [s.1.] Altas, 2010.

LANE, P. R.; MILESI-FERRETTI, G. M. The external wealth of nations: measures of foreign assets and liabilities for industrial and developing countries. Journal of international Economics, v. 55, n. 2, p. 263-294, 2001.

LOBÃO, J. F. Contágio financeiro entre mercados de ações de países desenvolvidos durante a crise do Brasil de 1999. p. 137-167, 1999.

MACEDO, M. A. DA S.; CASA NOVA, S. P. DE C.; DE ALMEIDA, K. Mapeamento e análise bibliométrica da utilização da Análise Envoltória de Dados (DEA) em estudos em contabilidade e administração. Contabilidade, Gestão e Governança, v. 12, n. 3, 2010.

PASQUARIELLO, P. The anatomy of financial crises: Evidence from the emerging ADR market. Journal of International Economics, v. 76, n. 2, p. 193-207, 2008.

PERICOLI, M.; SBRACIA, M. A Primer on Financial Contagion. Journal of Economic Surveys, v. 17, n. 4, p. 571-608, set. 2003.

PEROBELLI, F. F. C.; VIDAL, T. L.; SECURATO, J. R. Avaliando o efeito contágio entre economias durante crises financeiras. Estudos Econômicos (São Paulo), v. 43, n. 3, p. 557594, set. 2013.

PRATES, D. M.; CUNHA, A. M.; LÉLIS, M. T. C. O Brasil e a crise financeira global: avaliando os canais de transmissão nas contas externas. Revista de Economia Contemporânea, 
v. 15, n. 1, p. 62-91, abr. 2011.

ROSTAING, H. La bibliométrie et ses techniques. [s.1.] Sciences de la sociét Toulouse, 1996.

SCHEINKMAN, J. A.; XIONG, W. Overconfidence and speculative bubbles. Journal of political Economy, v. 111, n. 6, p. 1183-1220, 2003.

SCOPUS, S. Content coverage guide.Elsevier BV Amsterdam, Netherlands, , 2015.

STOVE, B.; TJOSTHEIM, D.; HUFTHAMMER, K. O. Using local Gaussian correlation in a nonlinear re-examination of financial contagion. Journal of Empirical Finance, v. 25, p. 62 82, 2014.

TORRES FILHO, E. T. Entendendo a crise do subprime. Visão do desenvolvimento, n. 44, p. $18,2008$.

VARTANIAN, P. R. Impactos do índice Dow Jones, commodities e câmbio sobre o Ibovespa: uma análise do efeito contágio. Revista de Administração Contemporânea, v. 16, n. 4, p. 608-627, ago. 2012.

XANTHOPOULOU, D. et al. Work engagement and financial returns: A diary study on the role of job and personal resources. Journal of Occupational and Organizational Psychology, v. 82, n. 1, p. 183-200, 2009. 